\title{
Information Uncertainty in Electricity Markets: Introducing Probabilistic Offers
}

\author{
Papakonstantinou, Athanasios; Pinson, Pierre
}

Published in:

IEEE Transactions on Power Systems

Link to article, DOI:

10.1109/TPWRS.2016.2540525

Publication date:

2016

Document Version

Peer reviewed version

Link back to DTU Orbit

Citation (APA):

Papakonstantinou, A., \& Pinson, P. (2016). Information Uncertainty in Electricity Markets: Introducing Probabilistic Offers. IEEE Transactions on Power Systems, 31(6), 5202 - 5203.

https://doi.org/10.1109/TPWRS.2016.2540525

\section{General rights}

Copyright and moral rights for the publications made accessible in the public portal are retained by the authors and/or other copyright owners and it is a condition of accessing publications that users recognise and abide by the legal requirements associated with these rights.

- Users may download and print one copy of any publication from the public portal for the purpose of private study or research.

- You may not further distribute the material or use it for any profit-making activity or commercial gain

- You may freely distribute the URL identifying the publication in the public portal

If you believe that this document breaches copyright please contact us providing details, and we will remove access to the work immediately and investigate your claim. 


\title{
Information Uncertainty in Electricity Markets: Introducing Probabilistic Offers
}

\author{
Athanasios Papakonstantinou, Member, IEEE, Pierre Pinson, Senior Member, IEEE.
}

\begin{abstract}
We propose a shift from the current paradigm of electricity markets treating stochastic producers similarly to conventional ones in terms of their offers. We argue that the producers' offers should be probabilistic to reflect the limited predictability of renewable energy generation, while we should design market mechanisms to accommodate such offers. We argue that the transition from deterministic offers is a natural next step in electricity markets, by analytically proving our proposal's equivalence with a two-price conventional market.

Index Terms -Electricity markets, renewable energy, imperfect information
\end{abstract}

\section{INTRODUCTION}

As generation from renewable sources of energy (RES) reaches grid parity, RES producers are asked to participate in electricity markets under the same rules as conventional generators. However, keeping the support mechanisms that were introduced to promote their development, has allowed them to exploit their new role by employing trading strategies that hedge their positions between day-ahead and real-time trading floors[1], [2]. It becomes clear that for RES to remain economically viable in the long-term, the regulation of their participation in electricity markets has to be challenged. In power systems dominated by RES, RES producers will have to be held accountable for the uncertainty they bring in power systems, by having the probabilistic estimates of their production evaluated. Naturally, electricity markets accommodating probabilistic offers can lead towards accountability.

In this context, we show analytically that an existing market mechanism using deterministic offers (i.e. a two-price balancing market) can be generalized to a probabilistic market where deterministic point forecasts are a specific case. We propose a probabilistic market based on an affine transformation of strictly proper scoring rules (i.e. Brier and Continuous Ranked Probability Scores). Such functions were designed to elicit accurate and precise probabilistic estimates from forecasters [3] and using them as the foundation of an electricity market achieves the same for the producers. This opens the door to appropriate valuation and use of the uncertainty information revealed through market participation.

\section{From a Deterministic to A Probabilistic ELECTRICITY MARKET}

We model conventional and stochastic power production as a possible realization $y$ of a random variable $Y$, scaled in

A. Papakonstantinou and P. Pinson are with the Department of Electrical Engineering of the Technical University of Denmark, Kgs. Lyngby, emails: \{athpapa,ppin\}@elektro.dtu.dk.

The authors are partly supported by the Danish Council for Strategic Research (DSF) through the project '5s-Future Electricity Markets', No. 12132636/DSF.

Manuscript received April 23, 2015
$[0,1]$. Let $Y$ follow a distribution with density $g(y)$ and $y_{0}$ denote the actual power production. Now, for conventional production $g$ is in-fact a scaled Dirac delta function i.e. all its values are equal to 0 except at $y_{0}$, with its integral over the real line equal to 1 . However, stochastic producers generate predictive distribution densities denoted as $f(y)$ to represent the probability distribution, $g(y)$. It is important to clarify that the stochastic producers' forecasted densities may not be equal to the distribution of the actual production i.e. $f \neq g$.

The energy dispatch model consists of a day-ahead market cleared at a price denoted by $\lambda^{\mathrm{DA}}$ followed by a real-time balancing market which settles energy deviations w.r.t the dayahead schedule. Under the two-price system deviations are priced differently depending on the sign of the imbalance; consequently the real-time clearing price $\lambda^{R T}$ is equal to $\lambda^{\mathrm{UP}}$ or $\lambda^{\mathrm{DN}}$ depending upon to positive or negative system balance. In a market with deterministic offers, the potential imbalance for producers is between their reported day-ahead offer $\widehat{y}$ (i.e. a point forecast) and the realized production $y_{0}$ estimated by $f(y)$. Following the process outlined in [1], the producer's revenue based on that imbalance is given by:

$$
P(\widehat{y})=\lambda^{\mathrm{DA}} y_{0}+ \begin{cases}\left(\lambda^{\mathrm{UP}}-\lambda^{\mathrm{DA}}\right)\left(y_{0}-\widehat{y}\right) & \text { if } y_{0} \leq \widehat{y} \\ \left(\lambda^{\mathrm{DN}}-\lambda^{\mathrm{DA}}\right)\left(y_{0}-\widehat{y}\right) & \text { if } y_{0}>\widehat{y}\end{cases}
$$

In this context, a risk neutral stochastic producer determines his offer $y^{*}$ in order to maximize expected revenue. Under the common practice in the literature, e.g. [1], [2], that the producer is a price-taker and therefore $\lambda^{\mathrm{DA}}$ is independent of $\widehat{y}$, the optimal offer is: $y^{*}=F^{-1}(\alpha) . F$ is the CDF of wind power production $y$ and $\alpha \leq 1$ and equal to $\Lambda^{\mathrm{DN}} /\left(\Lambda^{\mathrm{DN}}+\right.$ $\left.\Lambda^{\mathrm{UP}}\right)$, with $\Lambda^{\mathrm{UP}}=\lambda^{\mathrm{UP}}-\lambda^{\mathrm{DA}}$ and $\Lambda^{\mathrm{DN}}=\lambda^{\mathrm{DA}}-\lambda^{\mathrm{DN}}$ denoting the unit regulation cost for positive and negative imbalances. Given the above definitions of $\alpha, \Lambda^{\mathrm{UP}}$ and $\Lambda^{\mathrm{DN}}: 1-\alpha=$ $\Lambda^{\mathrm{UP}} /\left(\Lambda^{\mathrm{DN}}+\Lambda^{\mathrm{UP}}\right)$. We scale $P(\widehat{y})$ in Eq. (1) by multiplying by 2 and dividing by $\Lambda^{\mathrm{DN}}+\Lambda^{\mathrm{UP}}$. $P^{S}$ denotes the scaled quantity:

$$
\begin{aligned}
P^{S}(q) & =\frac{2 \lambda^{\mathrm{DA}} y_{0}}{\Lambda^{\mathrm{DN}}+\Lambda^{\mathrm{UP}}}+2\left(\alpha-\mathbb{I}\left\{y_{0} \leq q\right\}\right)\left(q-y_{0}\right) \\
& =\frac{2 \lambda^{\mathrm{DA}} y_{0}}{\Lambda^{\mathrm{DN}}+\Lambda^{\mathrm{UP}}}-\mathrm{QS}_{\alpha}\left(q, y_{0}\right)
\end{aligned}
$$

where $q$ is a quantile forecast at level $\alpha$ and $\mathrm{QS}_{\alpha}\left(q, y_{0}\right)$ is the Quantile Score (QS), a strictly proper scoring rule measuring in-sample goodness of fit and out-of-sample forecast performance [3]. According to [3] QS is equivalent to the Brier Score (BS), a strictly proper scoring rule used to verify predictions of the occurrence of a specific event, denoted by:

$$
\operatorname{BS}\left(p, y_{0}\right)=\left(p-\mathbb{I}\left\{y_{0} \leq y\right\}\right)^{2}
$$


where $p=F(y)$ is the probability of occurrence of a binary event $\{Y \leq y\}$ as reported by the forecaster. The predicted event is characterized by a threshold value $y$, which divides a real line in two intervals s.t. $I_{1}=(-\infty, y]$ and $I_{2}=(y, \infty)$. The event is said to occur if $y_{0} \leq y$, i.e. the indicator function $\mathbb{I}\left\{y_{0}<y\right\}$ is equal to 1 .

Based on the definitions of the scoring rules, we substitute the QS with the BS and reverse the transformation in Eq. (2). This yields the following expression for revenues:

$$
P\left(F, y_{0}\right)=\lambda^{\mathrm{DA}} y_{0}-\frac{\Lambda^{\mathrm{DN}}+\Lambda^{\mathrm{UP}}}{2}\left(F(y)-\mathbb{I}\left\{y_{0} \leq y\right\}\right)^{2}
$$

To this end, formulating a producer's revenue as a linear transformation of a strictly proper scoring rule shows that it is possible to design a market which accepts probabilistic offers without a complete overhaul of existing structures. In fact, for the stochastic producers opting to use deterministic offers or for the conventional producers and those offering perfect estimates (i.e. $\widehat{y}=y_{0}$ ), the Brier Score payment reverts to the current two-price payment.

\section{Evaluation of Probabilistic OfFERs through THE CONTINUOUS Ranked PRobability SCORE}

The design of an electricity market based on a linear transformation of the Continuous Ranked Probability Score (CRPS) allows the use of CDFs or more realistically a set of quantiles as market offers. Following [3] and [4] we construct the CRPS by calculating the average BS over all possible $y$ values, while taking note that the CRPS can be written as a kernel score [3]:

$$
\operatorname{CRPS}\left(F, y_{0}\right)=E_{F}\left|Y-y_{0}\right|-\frac{1}{2} E_{F}\left|Y-Y^{\prime}\right|
$$

where $Y$ and $Y^{\prime}$ are independent random variables of the same distribution $F$. The first part of Eq. (5) measures the divergence between predictive and actual distributions, while $1 / 2 E_{F}\left|Y-Y^{\prime}\right|$ is an information measure that coincides with the selectivity function denoted by: $U(F)=\int_{0}^{\infty} F(y)(1-$ $F(y)) d y$ [3], [5].

In the context of electricity markets the use of CRPS allows the information measure to be associated with the day-ahead stage of the mechanism, while divergence associates with real-time balancing. We calculate the average revenue $\bar{P}$ by integrating Eq. (4) with respect to the value of $y$ s.t.:

$$
\bar{P}\left(F, y_{0}\right)=\lambda^{\mathrm{DA}} y_{0}-\frac{\Lambda^{\mathrm{DN}}+\Lambda^{\mathrm{UP}}}{2} \operatorname{CRPS}\left(F, y_{0}\right)
$$

\section{A Probabilistic Electricity Market}

Based on the relation between the BS, the CRPS and the two-price system, we propose an electricity market which accepts probabilistic offers. Such offers reveal probabilistic information about future power production and may take the form of a CDF, quantiles, or parameters for known distributions. Both day-ahead and real-time markets are designed based on this type of offers to achieve the following objectives:

1) The day-ahead market holds them accountable for generating forecasts of low predictive value i.e. low precision or high dispersion.

2) The real-time market now additionally rewards or penalizes the stochastic producers based on the goodness of fit of the distributions and the quality of the estimate i.e. divergence from the realized production.

Regarding supply the market model is defined as follows:

\section{Day-ahead market:}

1) Each producer $i \in N$ submits his distribution-price offer $\left(F_{i}, c_{i}\right)$;

2) The market price $\lambda^{\mathrm{DA}}$ is determined based on the mean of the reported distribution following conventional market clearing rules;

3) Each producer receives the following payment,

$$
P_{i}^{\mathrm{DA}}=\lambda^{\mathrm{DA}} \bar{y}_{i}-\lambda^{\mathrm{DA}} U\left(F_{i}\right)
$$

\section{Real-time regulation market:}

1) Each stochastic producers generates outputs $y_{0_{i}}$ and up-regulation $\lambda^{\mathrm{UP}}$ or down-regulation $\lambda^{\mathrm{DN}}$ prices are determined as in the two-price system;

2) Each producer receives the following payment,

$$
\begin{aligned}
& P_{i}^{\mathrm{RT}}=\lambda^{\mathrm{DA}}\left(y_{0_{i}}-\bar{y}_{i}\right)+\lambda^{\mathrm{DA}} U\left(F_{i}\right) \\
& -1 / 2\left(\lambda^{\mathrm{UP}}-\lambda^{\mathrm{DN}}\right) \mathrm{BS}\left(F_{i}, y_{0_{i}}\right)
\end{aligned}
$$

Total Payment: Based on the realization of each producer's production $y_{0_{i}}$, the total revenue, is given by

$$
P\left(F, y_{0_{i}}\right)=\lambda^{\mathrm{DA}} y_{0_{i}}-1 / 2\left(\lambda^{\mathrm{UP}}-\lambda^{\mathrm{DN}}\right) \mathrm{BS}\left(F_{i}, y_{0_{i}}\right)
$$

Now, since $\overline{\mathrm{BS}}=\mathrm{CRPS}$, the revenue can be expressed as an affine transformation of the CPRS, given by Eq. (6), while also preserving the sign of the two-price payment. Therefore, the proposed market satisfies the required objectives of precision and accuracy, with the stochastic producers being held accountable for the uncertainty they introduce in the system. The proposed market motivates stochastic producers to reveal their true estimates, while conventional producers face no additional penalties given that there is no uncertainty in their offers.

\section{CONClusion}

This letter proves that submitting probabilistic offers in a day-ahead market can lead to the development of market mechanisms which can evaluate, and consequently reward or penalize, stochastic producers regarding the precision and accuracy of the estimates of their production. We show a straight forward process which can transform a deterministic market to a fully stochastic one through the utilization of strictly proper scoring rules. The proposed market structure can be viewed as the generalization of the two-price balancing market and therefore shares its favorable properties.

\section{REFERENCES}

[1] J. M. Morales, A. J. Conejo, H. Madsen, P. Pinson, and M. Zugno, Integrating Renewables in Electricity Markets. Springer, 2013.

[2] C. J. Dent, J. W. Bialek, and B. F. Hobbs, "Opportunity Cost Bidding by Wind Generators in Forward Markets: Analytical Results," IEEE Transactions on Power Systems, vol. 26, no. 3, pp. 1600-1608, 2011.

[3] T. Gneiting and A. E. Raftery, "Strictly proper scoring rules, prediction, and estimation," Journal of the American Statistical Association, vol. 102, no. 477, pp. 359-378, 2007.

[4] H. Hersbach, "Decomposition of the continuous ranked probability score for ensemble prediction systems," Weather and Forecasting, vol. 15, pp. 559-570, 2000.

[5] G. Matheron, "The selectivity of the distributions and the second principle of geostatistics," in Geostatistics for Natural Resources Characterization, G. Verly et al., Ed. Springer Netherlands, 1984, pp. 421-433. 